\title{
Growth hormone receptor controls adipogenic differentiation of chicken bone marrow mesenchymal stem cells by affecting mitochondrial function
}

\section{Changbin Zhao}

South China Agricultural University

\section{Bowen Hu}

South China Agricultural University

Haohui Wei

South China Agricultural University

Wen Luo

South China Agricultural University

Yongxia Zhao

South China Agricultural University

Jinping Liang

South China Agricultural University

Qinghua Nie

South China Agricultural University

Qingbin Luo

South China Agricultural University

Dexiang Zhang

South China Agricultural University

Xiquan Zhang

South China Agricultural University https://orcid.org/0000-0002-7940-1303

Hongmei Li ( $\nabla$ hongmeili@scau.edu.cn )

South China Agricultural University https://orcid.org/0000-0002-2013-1679

\section{Research}

Keywords: Adipogenic differentiation, Bone mesenchymal stem cells, Growth hormone receptor, Mitochondrial function, Sex-linked dwarf chickens

Posted Date: September 24th, 2021 
DOl: https://doi.org/10.21203/rs.3.rs-915843/v1

License: (c) (1) This work is licensed under a Creative Commons Attribution 4.0 International License. Read Full License 


\section{Abstract \\ Background}

Growth hormone receptor (GHR), a member of the type I cytokine receptor family, activates several downstream signaling pathways after binding to growth hormone $(\mathrm{GH})$ to regulate cell growth and development. However, in poultry, the relations among the GHR gene, mitochondrial function, and adipocyte differentiation remain unclear.

\section{Methods}

In this study, we used sex-linked dwarf (SLD) chickens and normal chickens as research objects and overexpression or knockdown of GHR in bone mesenchymal stem cells (BMSCs) at the stage of adipogenic differentiation, to investigate the effects of the GHR gene on mitochondrial biogenesis, mitochondrial function and adipogenic differentiation of BMSCs.

\section{Results}

Yellow bone marrow in SLD chickens was higher than that in normal chickens, and lipid droplets and triglyceride content also increased in SLD bone marrow tissue. Mitochondrial biogenesis and function in BMSCs were compared between SLD and normal chickens, mitochondrial membrane potential decreased, mitochondrial ROS (mtROS) and ATP contents increased. Expression of genes associated with mitochondrial biogenesis and function, including ND1, ND2, CYTB, COX1, COX2, ATP6, ATP8, PGC1a, NRF1, and TFAM, also increased in BMSCs of SLD chickens. In addition, GHR was overexpressed and knocked down in chicken BMSCs to verify results in vitro. Overexpression of GHR increased mitochondrial membrane potential but decreased mtROS and ATP contents, oxidative phosphorylation complex enzyme activity, and mitochondrial number. Expression of genes associated with mitochondrial biogenesis and function was also repressed during adipogenic differentiation in chicken BMSCs. After overexpression of $G H R$, changes in adipogenic differentiation capacity of chicken BMSCs were also examined, and expression of genes associated with adipogenic differentiation, including $P P A R \gamma, C / E B P a$, and $C / E B P \beta$, decreased. Triglyceride production and number of lipid droplets (oil red $O$ staining) also decreased after $G H R$ overexpression. With knockdown of GHR, opposite results were observed.

\section{Conclusions}

GHR inhibited adipogenic differentiation of chicken BMSCs by suppressing mitochondrial function. This suppression might explain the clinical manifestation of severe fat deposition in SLD chickens.

\section{Background}


Growth hormone $(\mathrm{GH})$, a peptide hormone regulated by the hypothalamus and secreted by the pituitary gland, binds to cell surface growth hormone receptors to regulate metabolic processes and growth and development[1]. Growth hormone receptor (GHR), a member of the type I cytokine receptor family, is the key receptor transmembrane protein in the $G H-G H R$ axis. The GHR protein has three primary domains: extracellular, single-pass transmembrane, and cytoplasmic intracellular [2]. In sex-linked dwarf (SLD) chickens, normal protein functions are prevented because of exon mutations in the GHR gene, compared with normal chickens [3]. Sex-linked dwarf chickens are short, weigh only $60-70 \%$ of normal chickens, and have higher feed utilization than normal but also more severe fat deposition [4]. The molecular mechanisms responsible for the SLD phenotype are not clearly understood.

$G H$ can regulate mitochondrial respiration by binding to the Box1 region of the GHR gene [5]. In GHR knockout (GHRKO) mice, mitochondrial function and antioxidant capacity increase [6]. Mitochondrial biogenesis maintains mitochondrial homeostasis and function by producing new mitochondria [7], and in GHRKO mice, key regulators of mitochondrial biogenesis increase in liver, kidney, and skeletal muscle [8, 9]. However, those results conflict with those of other reports. Mitochondrial function is severely impaired in osteoblasts and fibroblasts of GHRKO mice [10], and deletion of GHR function impairs mitochondrial function in chicken skeletal muscle and DF-1 cells [11]. Such conflicting results suggest that the effects of $G H R$ on mitochondria vary by species and tissue.

Bone mesenchymal stem cells (BMSCs), also known as bone marrow-derived mesenchymal stem cells, are multipotent stromal cells with self-renewing ability and multilineage differentiation [12]. They can differentiate into many different types of cells, including adipocytes, osteoblasts, chondrocytes, myocytes, and neurons [13]. Most current research on BMSCs focuses on both adipogenesis and osteogenesis. Adipogenic and osteogenic differentiation of BMSCs is regulated by multiple signaling and transcription factors [14]. In general, adipogenic differentiation and osteogenic differentiation in BMSCs are mutually exclusive, with stimulation of osteogenesis suppressing adipogenesis and vice versa [15]. When expression of peroxisome proliferator-activated receptor-y (PPARY) is suppressed, the ability of BMSCs to differentiate toward adipogenesis diminishes, whereas the ability to differentiate toward osteogenesis increases [16]. In GHRKO mice, expression of genes associated with adipogenic differentiation increases in mesenchymal stem cells (MSCs), and the ability to differentiate toward adipogenesis also increases [17]. However, specific mechanisms of the $G H-G H R$ axis and adipogenic differentiation of BMSCs are unclear.

During proliferation of MSCs, the primary metabolic mode is glycolysis, whereas during their differentiation, the primary mode shifts from glycolysis to mitochondrial-based oxidative phosphorylation [18]. Reactive oxygen species (ROS) produced by mitochondrial complex III are required to initiate adipocyte differentiation [19]. A key regulator of mitochondrial biogenesis, PGC1a, promotes adipogenic differentiation and inhibits osteogenic differentiation in immortalized human MSCs [20]. Mitochondria are highly dynamic organelles that can be rapidly restructured to meet metabolic demands in a timely manner [21]. Thus, mitochondria are essential in adipogenic differentiation of BMSCs. 
In SLD chickens, liver mitochondrial function declines compared with that in normal chickens [22]. Therefore, SLD and normal chickens were compared in this study, and overexpression and knockdown of GHR in BMSCs were used to determine the effects of GHR on mitochondrial function and adipogenic differentiation of chicken BMSCs.

\section{Methods}

\section{Ethics statement}

All animal experiments were performed according to the protocols approved by the South China Agriculture University Institutional Animal Care and Use Committee (approval number SCAU\#0017). All animal procedures followed the regulations and guidelines established by this committee and minimized the suffering of animals.

\section{Chickens}

For in vivo experiments, the Guangdong Wenshi Southern Poultry Breeding Co., Ltd. (Guangzhou, China), provided 21-d-old yellow-feather chickens. To isolate bone BMSCs, the Yuhe Agriculture and Animal Husbandry Co., Ltd. (Guangzhou, China), provided 3-d-old chickens.

The 21-d-old yellow-feather chickens included 15 SLD chickens and 15 normal chickens. To explore molecular mechanisms of GHR in vivo and determine the cause of fatty deposits in SLD chickens, mitochondrial function, mitochondrial biogenesis, and adipogenic differentiation in chicken BMSCs were examined in those chickens. The 3-d-old normal chickens were only used to isolate BMSCs, and the BMSCs were used to study GHR effects on mitochondria and adipogenic differentiation in vitro.

\section{Paraffin sections and hematoxylin and eosin staining}

The epiphyses of thighbone from the 21-d-old SLD and normal chickens were fixed with $10 \%$ neutral formalin for $5 \mathrm{~d}$ and then immersed in a hydrochloric acid/formic acid working solution to complete decalcification. After decalcification, samples were dehydrated in alcohol and transformed into a transparent state using xylene. After completing the transparency step, samples were soaked in wax and embedded in paraffin. A paraffin sectioning machine cut 7 to $10-\mu \mathrm{m}$-thick sections, which were stained with hematoxylin and eosin.

\section{Frozen sections and oil red staining}

Epiphyseal parts of femurs of the 21-d-old SLD and normal chickens were cut off and soaked in $4 \%$ paraformaldehyde for $48 \mathrm{~h}$ and then switched to decalcification solution for $30 \mathrm{~d}$, with the solution changed every two days. After decalcification, tissues were placed in a $15 \%$ sucrose solution in a refrigerator at $4{ }^{\circ} \mathrm{C}$ to dehydrate and sink and then were transferred to a $30 \%$ sucrose solution at $4{ }^{\circ} \mathrm{C}$ to dehydrate and sink. Dehydrated tissue was placed cut side up on a sample tray and surrounded by drops of OCT embedding agent (Servicebio, Wuhan, China). The tray was placed on the quick-freeze table of a 
frozen sectioning machine, and the samples were sectioned after the OCT whitened and hardened. Section thickness was 8 to $10 \mu \mathrm{m}$. After sectioning, tissue sections were fixed on a slide and stained with oil red and hematoxylin.

\section{Detection of triglyceride}

Triglyceride was measured using a Triglyceride Assay Kit (Nanjing Jiancheng, Nanjing, China) according to the manufacturer's protocol. Triglyceride was measured at $510 \mathrm{~nm}$, and absorbance was determined using a Fluorescence/Multi-Detection Microplate Reader (Bio-Tek, Winooski, USA) according to the manufacturer's protocol. Data were normalized to the control group and expressed as a percentage of the control.

\section{Reverse-transcription quantitative PCR}

RNA was extracted from tissues or cells using RNAiso reagent (Takara, Shiga, Japan) according to the manufacturer's protocol. Concentration of RNA samples and optical density (OD) value of 260/280 were detected using a Nanodrop 2000c spectrophotometer (Thermo, Waltham, USA). Samples were stored at $-80^{\circ} \mathrm{C}$ for later use. For reverse-transcription quantitative PCR (RT-qPCR), cDNA was synthesized using MonScript ${ }^{\text {TM }}$ RTIII All-in-One Mix with dsDNase (Monad Co., Ltd., Guangzhou, China). ChamQ Universal SYBR qPCR Master Mix (Vazyme, Guangzhou, China) was used in RT-qPCRs run on a Bio-Rad CFX96 Real-Time Detection instrument (Bio-Rad, Hercules, USA) according to the manufacturer's protocol. The reaction procedure included initial denaturation at $95^{\circ} \mathrm{C}$ for $3 \mathrm{~min}$, followed by denaturation at $95^{\circ} \mathrm{C}$ for $10 \mathrm{~s}$ and annealing at $60^{\circ} \mathrm{C}$ for $30 \mathrm{~s}$, for a total of 40 cycles. At the end of the cycle, the dissolution curve was analyzed, and the detection temperature was $65^{\circ} \mathrm{C}$ to $95^{\circ} \mathrm{C}$. Relative gene expression was measured using RT-qPCR twice for each reaction, and $\beta$-actin was used as the control. The primers used in RT-qPCR are listed in Table 1.

\section{Extraction of chicken bone mesenchymal stem cells and cell culture}

Bone mesenchymal stem cells were extracted using the appropriate separation kits (TBD science, Tianjin, China) following the manufacturer's protocol.

Bone mesenchymal stem cells from 21-d-old SLD and normal chickens were extracted by cell separation kits and cultured in vitro to the appropriate density (the first generation). Then, assays were conducted on mitochondrial function and related gene expression and protein levels.

Bone mesenchymal stem cells from 3-d-old normal chickens were extracted by cell separation kits and were cultured in vitro and passaged to the third generation. Overexpression and knockdown of GHR were to explore the effects of GHR on mitochondrial and adipogenic differentiation in chicken BMSCs.

Bone mesenchymal stem cells were cultured in Gibco Dulbecco's Modified Eagle Medium (DMEM): F-12 (Gibco, Waltham, USA) with 10\% fetal bovine serum (FBS) (Gibco) and 1\% penicillin/streptomycin (Gibco). All cells were cultured at $37^{\circ} \mathrm{C}$ in a $5 \% \mathrm{CO}_{2}$ humified atmosphere. 


\section{Induction of adipogenic differentiation}

Bone mesenchymal stem cells were seeded into 6-well plates at $1.25 \times 10^{5}$ cells per $\mathrm{cm}^{2}$. Bone mesenchymal stem cells were induced with adipogenic medium containing DMEM/F12 (10\% FBS), 0.5 mM 3-isobutyl-1-methylxanthine (Sigma-Aldrich, Darmstadt, Germany), $1 \mu \mathrm{M}$ dexamethasone (SigmaAldrich), $10 \mu \mathrm{g} / \mathrm{mL}$ insulin (Sigma-Aldrich), and $200 \mu \mathrm{M}$ indomethacin (Sigma-Aldrich). The medium was replaced every $2 \mathrm{~d}$ for $6 \mathrm{~d}$.

\section{Plasmid construction, small interfering RNA, and transfection}

Third generation BMSCs were plated onto 6-well plates, and transfection began when the density reached approximately $80 \%$. After $6 \mathrm{~h}$ of transfection, the DMEM/F12 medium was changed to adipogenic induction medium to induce adipogenic differentiation of BMSCs.

GeneCreate (Wuhan, China) synthesized the plasmid pcDNA3.1-GHR. Plasmid transfection was performed using Lipofectamine 3000 reagent (Invitrogen, Waltham, USA) following the manufacturer's protocol, and nucleic acids were diluted in OPTI-MEM (Gibco). All cells were analyzed $72 \mathrm{~h}$ after transfection.

Guangzhou RiboBio (Guangzhou, China) synthesized small interfering RNAs (siRNA) used for GHR knockdown. In preliminary experiments, four siRNAs were designed to interfere with $G H R$, and the si$G H R$ with the highest interference efficiency was used. The siRNA sequence is provided in Table 2. The siGHR sequence was transfected in BMSCs to a final concentration of $100 \mathrm{nM}$ using Lipofectamine 3000 reagent (Invitrogen, USA) according to the manufacturer's protocol. Cells were analyzed at $72 \mathrm{~h}$ after transfection.

\section{Detection of reactive oxygen species}

Production of ROS in mitochondria was measured using an ROS assay kit (Beyotime, Shanghai, China) according to the manufacturer's protocol. Dichlorofluorescein (DCF) fluorescence was determined using a Fluorescence/Multi-Detection Microplate Reader (Bio-Tek). Data were normalized to the control group and are expressed as a percentage of the control.

\section{Detection of ATP content}

ATP levels were measured using an ATP assay kit (Beyotime) according to the manufacturer's protocol. A Fluorescence/Multi-Detection Microplate Reader (BioTek) was used to determine ATP levels. Data were normalized to the control group and are expressed as a percentage of the control.

\section{Detection of mitochondrial membrane potential}

Mitochondrial membrane potential $(\Delta \Psi \mathrm{m})$ was measured using a JC-1 kit (Beyotime) according to the manufacturer's protocol. Mitochondria were fixed with JC-1, and after cells were incubated with JC-1 for 
20 min at $37^{\circ} \mathrm{C}$, fluorescence was determined using a Fluorescence/Multi-Detection Microplate Reader (Bio-Tek). Rotenone, $10 \mu \mathrm{mol} / \mathrm{L}$, was used as a standard inhibitor of $\Delta \Psi \mathrm{m}$. Data (the ratio of aggregated and monomeric JC-1) were normalized to the control group and are expressed as a percentage of the control.

\section{Detection of enzymatic activity of mitochondrial oxidative phosphorylation complexes}

Commercial assay kits (Solarbio, Beijing, China) were used to measure enzyme activity of mitochondrial oxidative phosphorylation (OXPHOS) complexes in BMSCs according to the manufacturer's protocol. Complex I enzyme activity was determined by the change in absorbance of NADH at $340 \mathrm{~nm}$. Complex II enzyme activity was determined by the change in absorbance of 2,6-dichlorophenol indophenol at 600 $\mathrm{nm}$. Enzyme activity of complex III and complex IV was determined by the change in absorbance of reduced cytochrome $\mathrm{c}$ at $550 \mathrm{~nm}$. Absorbance was determined using a Fluorescence/Multi-Detection Microplate Reader (Bio-Tek). Data were normalized to the control group and are expressed as a percentage of the control.

\section{Mito-tracker green staining and Hoechst 33342 staining}

Mito-tracker green staining and Hoechst 33342 staining were used to label mitochondria and nuclei in BMSCs, respectively. At $72 \mathrm{~h}$ after transfection, cells were washed twice with phosphate buffered saline (PBS) and incubated with Mito-tracker green (Beyotime) for $30 \mathrm{~min}$. Cells were then suspended in PBS, and $10 \mu \mathrm{L}$ of Hoechst 33342 dye was added (Beyotime). After washing twice with PBS, a fluorescence microscope (Nikon TE2000-U, Tokyo, Japan) was used to capture five randomly selected fields that were analyzed with NIS-Elements software.

\section{Oil red 0 staining and quantification}

Bone mesenchymal stem cells were seeded into 6-well culture plates. After transfection and differentiation for $5 \mathrm{~d}$, differentiated BMSCs were washed with PBS and then fixed with $4 \%$ formaldehyde for 30 min. Differentiated BMSCs were dyed with oil red O working solution (BBI, Shanghai, China) for 60 min at room temperature and then washed three times with PBS, according to the manufacturer's specification. After washing, a fluorescence inverted light microscope (Leica DMi8, Wetzlar, Germany) was used to capture images. At the end, stain in cells was extracted by isopropanol and absorbance was measured at $510 \mathrm{~nm}$ with a Fluorescence/Multi-Detection Microplate Reader (Bio-Tek).

\section{Western blot analysis}

Radio-immune precipitation assay buffer (Beyotime) with phenylmethane sulfonyl fluoride protease inhibitor (Beyotime) was used to lyse tissue and cellular proteins. The homogenate was centrifuged at $13,000 \times g$ for $10 \mathrm{~min}$ at $4^{\circ} \mathrm{C}$. The supernatant was collected, and protein concentration was determined immediately using a bicinchoninic acid assay protein quantification kit (Beyotime). Proteins were separated in $10 \%$ sodium dodecyl sulfate-polyacrylamide gel electrophoresis, transferred onto a polyvinylidene difluoride membrane, and then probed with antibodies following standard procedures. 
The following antibodies and their dilutions were used in western blot: mouse anti-PGC1 alpha antibody (1C1B2, 1:5,000; Proteintech, Rosemont, USA), rabbit anti-NRF1 antibody (AF7620, 1:1,000; Beyotime), rabbit anti-TOMM20 antibody (AF1717, 1:1,000; Beyotime), rabbit anti-PPAR gamma polyclonal antibody (bs-0530R, 1:1,000; Bioss, Beijing, China), rabbit anti-CEBP alpha polyclonal antibody (bs-24540R, 1:1,000; Bioss), rabbit anti-beta-actin antibody (bs-0061R, 1:5,000; Bioss), goat anti-rabbit IgG-HRP (BS13278, 1:10,000; Bioworld, Minnesota, USA), and goat anti-mouse IgG-HRP (BS12478, 1:10,000; Bioworld).

\section{Statistical analyses}

All experiments were performed at least three times. Data are presented as the mean \pm standard error of the mean (SEM). Statistical analyses were performed using Student's $t$-test, with statistical significance indicated as $* P<0.05,{ }^{\star *} P<0.01$, and ${ }^{* \star *} P<0.001$.

\section{Results}

\section{Fat deposition and differentiation of bone mesenchymal stem cells in bone marrow tissue of sex-linked dwarf and normal chickens}

Hematoxylin and eosin staining of bone marrow tissues from 21-d-old SLD and normal chickens showed the percentage of fat in bone marrow tissues of SLD chickens was significantly higher than that of normal chickens (Fig. 1a-c). Oil red O staining on bone marrow tissues of SLD and normal chickens showed the percentage of lipid droplets in bone marrow tissues of SLD chickens was higher than that of normal chickens (Fig. 1d-f). Furthermore, triglyceride content in the bone marrow tissue of SLD chickens was highly significantly higher than that of normal chickens (Fig. 1g). To investigate whether the cause of this phenotype in SLD chickens was due to differentiation of BMSCs, expression of marker genes of adipogenic differentiation in BMSCs of SLD and normal chickens was examined. Expression of the marker genes PPARY, C/EBPa, and C/EBP $\beta$ increased significantly in SLD chickens (Fig. $1 \mathrm{~h}$ ). The results were similar in protein level assays (Fig. 1i). Thus, fat deposition in the bone marrow tissue of SLD chickens was much more severe than that of normal chickens. This result might be due to differentiation of BMSCs in SLD chickens.

\section{Mitochondrial function in bone mesenchymal stem cells of sex-linked dwarf and normal chickens}

Mitochondria can synthesize ATP through oxidative phosphorylation to provide a major source of energy for adipogenic differentiation of MSCs [23]. Therefore, we speculated that mitochondria have an important role in the production of lipids in bone marrow tissue. The mRNA levels of mtDNA-encoded OXPHOS-related and mitochondrial biogenesis-related genes in BMSCs of SLD and normal chickens were measured by RT-qPCR. Genes included ND1, ND2, CYTB, COX1, COX2, ATP6, ATP8, PGC1a, NRF1, and TFAM. Protein levels of PGC1a, NRF1, and TOMM20 in BMSCs of SLD and normal chickens were measured by western blot. The mRNA expression of genes was elevated significantly in BMSCs of SLD chickens (Fig. 2a, b). Protein levels of PGC1a, NRF1, and TOMM20 also increased in SLD chickens 
(Fig. 2c). Furthermore, to indicate mitochondrial function, $\Delta \Psi \mathrm{m}$, ROS production, and ATP content were examined. Compared with normal chickens, $\Delta \Psi \mathrm{m}$ decreased and ROS production and ATP content increased in SLD chickens (Fig. 2d-f). Therefore, in SLD chickens, changes in mitochondrial function might affect adipogenic differentiation of BMSCs and thus adipogenesis.

\section{Effects of overexpression and knockdown of growth hormone receptor on mitochondrial function in chicken bone mesenchymal stem cells}

To investigate the effect of GHR on mitochondrial function, BMSCs were isolated and cultured in vitro, and then, GHR was overexpressed or knocked down. Efficiency of GHR overexpression and knockdown efficiency in chicken BMSCs differentiated for $72 \mathrm{~h}$ was examined by RT-qPCR. Compared with the control group, GHR expression was significantly up-regulated after transfection with the vector of GHR (Fig. 3a) and significantly down-regulated after transfection with si-GHR (Fig. 3b). Similar to in vivo experiments, mitochondrial function was examined after overexpression and knockdown of GHR in differentiated BMSCs. After overexpression of GHR in BMSCs, mRNA expression of mtDNA-encoded OXPHOS-related and mitochondrial biogenesis-related genes decreased (Fig. 3c, e). Protein levels of PGC1a, NRF1, and TOMM20 showed similar results (Fig. $3 \mathrm{~g}$ ). In addition, $\Delta \Psi \mathrm{m}$ increased, and ROS production and ATP content decreased (Fig. 3i, k, m). Opposite results were observed after knockdown of GHR (Fig. 3d, f, h, j, I, n). These results suggested that GHR repressed mitochondrial function during adipogenic differentiation in chicken BMSCs.

\section{Effects of overexpression and knockdown of growth hormone receptor on enzymatic activity of OXPHOS complexes in chicken bone mesenchymal stem cells}

To further investigate the effects of GHR on mitochondrial function in chicken BMSCs during adipogenic differentiation, enzyme activity of mitochondrial OXPHOS complexes was examined in differentiated BMSCs after overexpression and knockdown of GHR. Enzymatic activities of OXPHOS complexes I, II, III, and IV decreased significantly in chicken BMSCs after overexpression of GHR (Fig. 4a-d), whereas after GHR knockdown, enzymatic activities increased significantly (Fig. 4e-h). Overall, the results indicated that GHR repressed mitochondrial function by suppressing enzymatic activity of OXPHOS complexes in chicken BMSCs during adipogenic differentiation.

\section{Effects of overexpression and knockdown of growth hormone receptor on mitochondrial number and quality in chicken bone mesenchymal stem cells}

Mitochondrial biogenesis increases the number of mitochondria to meet intracellular energy requirements. Therefore, the effect of GHR on mitochondrial function was explored by assaying the number of mitochondria. Mito-tracker staining was used to label mitochondria, with fluorescence intensity representing mitochondrial quantity. After overexpression of GHR in BMSCs, fluorescence intensity weakened, and the number of mitochondria decreased (Fig. 5a, C). After knockdown of GHR, fluorescence intensity strengthened, and the number of mitochondria increased (Fig. 5b, d). Thus, in 
addition to repressing mitochondrial function, GHR reduced mitochondrial number and quality by repressing mitochondrial biogenesis during adipogenic differentiation of chicken BMSCs.

\section{Effects of overexpression and knockdown of growth hormone receptor on adipogenic differentiation of chicken bone mesenchymal stem cells}

Finally, GHR was overexpressed and knocked down in chicken BMSCs to investigate the effects of GHR on adipogenic differentiation. Adipogenic differentiation was induced in BMSCs, and expression of associated genes was detected by RT-qPCR. The genes were PPARY, $C / E B P a$, and $C / E B P \beta$. Protein levels of PPARY and C/EBPa in chicken BMSCs differentiated for $72 \mathrm{~h}$ were measured simultaneously by western blot. Expression of adipogenic differentiation-related genes was significantly down-regulated after overexpression of GHR (Fig. 6a), whereas after knockdown of GHR, expression was significantly upregulated (Fig. 6C). Protein levels of PPARY and C/EBPa in chicken BMSCs showed similar results (Fig. 6b, d). Furthermore, the oil red $\mathrm{O}$ test was used to measure lipid droplet content in chicken BMSCs differentiated for $5 \mathrm{~d}$ after overexpression and knockdown or GHR. Overexpression of GHR depressed the lipid droplet depot in BMSCs, whereas knockdown had the opposite effect (Fig. 6e-h). In addition, overexpression of GHR repressed triglyceride production in BMSCs (Fig. 6i), whereas knockdown of GHR (Fig. 6j) produced opposite results. Thus, GHR repressed fat deposition in chickens by inhibiting adipogenic differentiation of chicken BMSCs.

\section{Discussion}

Since their discovery in 1940, research on SLD chickens has been uninterrupted. Mutation of the GHR gene in SLD chickens interferes with binding of GH to GHR [24], and therefore, SLD chickens are a specific animal model for mutation of the GHR gene [25]. Fat deposition is more severe in SLD chickens than in normal chickens [4]. In a previous study, compared with normal chickens, red bone marrow was severely depleted and replaced by yellow bone marrow in 7-week-old SLD chickens [26]. It was hypothesized that the SLD phenotype was due to a functional deletion of the GHR gene. Therefore, in this study, the relation between GHR and adipogenic differentiation of BMSCs was explored.

In this study, fat deposition in bone marrow tissue of 21-d-old SLD chickens was greater than that in normal chickens, consistent with previous findings [26]. In addition, triglyceride content of bone marrow tissue in SLD chickens was twice as high as that in normal chickens, consistent with fat deposition. Fat in bone marrow tissue is primarily derived from adipogenic differentiation of MSCs [27]. The balance between adipose and bone tissues in bone marrow tissue is maintained primarily by two types of MSC differentiation: adipogenic and osteogenic [28]. When adipogenic differentiation of MSCs increases, osteogenic differentiation is relatively weakened, resulting in fat deposition [29]. Therefore, it was hypothesized that the more severe fat deposition in SLD bone marrow tissue was due to increased adipogenic differentiation capacity of BMSCs in SLD chickens because of deficiency in normal GHR gene function. To test the hypothesis, BMSCs were extracted from SLD and normal chickens, and differences in expression of genes associated with adipogenic differentiation in the two groups of cells were 
examined. Expression of PPARY, C/EBPa, and $C / E B P \beta$ in BMSCs was significantly higher in SLD chickens than in normal chickens. PPARY, as the predominant transcription factor in adipocyte differentiation, also plays an important role in adipogenic differentiation of BMSCs. In one study, addition of the PPARY agonist rosiglitazone activated adipogenic differentiation of mouse BMSCs [30]. During adipogenic differentiation of MSCs, $C / E B P \beta, P P A R \gamma$, and $C / E B P a$ are sequentially activated [31].

An increasing number of studies show that regulation of mitochondrial dynamics and function is critical for successful differentiation of MSCs. Adipogenic differentiation of MSCs is accompanied by changes in the mitochondrial phenotype, including increased mitochondrial biogenesis and abundance of OXPHOS complexes [32]. Therefore, mitochondrial function of BMSCs from SLD and normal chickens was examined by using ATP content, ROS, and $\triangle \Psi \mathrm{m}$ assays. The ATP content was higher in the BMSCs of SLD chickens, indicating that deficiency in GHR function led to an increase in mitochondrial oxidative phosphorylation capacity and therefore production of ATP. Those changes provided the necessary conditions for adipogenic differentiation of BMSCs. Reactive oxygen species are produced by the OXPHOS pathway associated with energy production in mitochondria [33]. Only unregulated levels of ROS are harmful, whereas regulated ROS production is needed for essential signaling pathways that regulate cell functions [34]. Production of ROS by mitochondrial complex III is required to activate adipogenic differentiation of MSCs, and ROS levels increase during adipogenesis induction in MSCs [19]. In this study, ROS production was greater in BMSCs of SLD chickens than in those of normal chickens. Reactive oxygen species promote lipid accumulation in human adipose stromal cells undergoing adipogenesis [35]. Therefore, increases in ROS may be one factor that stimulates differentiation of BMSCs toward adipogenesis. Notably, $\Delta \Psi \mathrm{m}$ decreased in BMSCs of SLD chickens compared with that in normal chickens. In general, the higher $\Delta \Psi_{\mathrm{m}}$ is, the greater the energy capacity of the inner mitochondrial membrane and the higher the amount of ATP synthesis [36]. However, maintaining excessively high or excessively low $\Delta \Psi_{\mathrm{m}}$ can be harmful to mitochondria and cells $[37,38]$. A decrease in $\Delta \Psi_{\mathrm{m}}$ is presumed to be due to the effect of "mild uncoupling of mitochondria", which ensures the supply of ATP while appropriately lowering the $\triangle \Psi \mathrm{m}$ in response to damage caused by elevated ROS [39]. In addition, expression of mitochondrial genes encoding OXPHOS, including ND1, ND2, CYTB, COX1, COX2, ATP6, and $A T P 8$, and genes related to mitochondrial biogenesis, including PGC1a, NRF1, and TFAM, were upregulated in BMSCs of SLD chickens compared with those in normal chickens. Levels of the mitochondrial membrane protein TOMM20 and expression of genes associated with mitochondrial biogenesis (PGC1a, NRF1) also increased. The results indicated that increases in mitochondrial function in BMSCs of SLD chickens were due to the absence of GHR function. Therefore, changes in mitochondria in BMSCs of SLD chickens may affect adipogenic differentiation of BMSCs and ultimately increase fat deposition in SLD chickens.

To further confirm the conjecture on the mechanism of fat deposition in bone marrow tissue of SLD chickens, BMSCs were extracted from chickens for cellular verification. Expression of mitochondrial genes encoding OXPHOS and genes associated with mitochondrial biogenesis was examined after overexpression and knockdown of GHR in BMSCs. The results were consistent with those obtained in vivo. When GHR was knocked down in chickens BMSCs, $\Delta \Psi_{\mathrm{m}}$ decreased, ROS production and ATP 
content increased, and protein levels of PGC1a, NRF1, and TOMM20 was enhanced. The opposite result after overexpression of $G H R$. Collectively, the results suggest that GHR inhibits mitochondrial function during adipogenic differentiation in chicken BMSCs. This conclusion is also supported by enzymatic activity of complexes I, II, III, and IV after overexpression and knockdown of GHR. Enzymatic activity of complexes I, II, III, and IV was enhanced after knockdown of GHR. The opposite result after overexpression of GHR. Complexes I, II, III, and IV are important components of the mitochondrial electron transport chain and are involved in the adipogenic differentiation of BMSCs through mitochondrial oxidative phosphorylation. In one study, inhibition of the mitochondrial electron transport chain suppressed adipogenic differentiation of MSCs [23]. Mitochondrial biogenesis is regulation of the number of mitochondria through mitochondrial self-renewal in response to energy demands triggered by developmental signals and environmental stressors [40]. Mitochondrial biogenesis increases during adipogenic differentiation of MSCs [23]. In immortalized human MSCs, overexpression of PGC-1a increases mitochondrial function and biogenesis and promotes adipogenic differentiation of MSCs [41]. TFAM can bind to the mitochondrial light strand promoter and functions in mitochondrial transcription regulation [41], and knockdown of TFAM in MSCs inhibits adipogenic differentiation [23]. Furthermore, Mito-tracker staining validated the effect of GHR on mitochondrial biogenesis. The number of mitochondria decreased after overexpression of GHR, indicating that GHR inhibited mitochondrial biogenesis. The opposite result was observed after knockdown of $G H R$.

In addition, whether GHR inhibited adipogenic differentiation of chicken BMSCs in vitro was investigated. After overexpression of GHR in chicken BMSCs, expression of differentiation-related genes, including $P P A R Y, C / E B P a$, and $C / E B P \beta$, was repressed and lipid droplet production and triglyceride levels decreased. Notably, PPARY determines the direction of adipogenic differentiation of MSCs [42]. With knockdown of GHR, opposite results were obtained. Thus, GHR can inhibit adipogenic differentiation of chicken BMSCs.

\section{Conclusions}

Sex-linked dwarf chickens had severe fat deposition in bone marrow tissue than normal chickens. Increased adipogenic differentiation of BMSCs in SLD chickens was associated with increases in mitochondrial biogenesis and function and expression of genes related to differentiation. After overexpression of GHR in chicken BMSCs, mitochondrial function and adipogenic differentiation of BMSCs were repressed. The opposite results were observed after knockdown of GHR. Therefore, GHR inhibits excessive adipogenic differentiation of chicken BMSCs by repressing mitochondrial function.

\section{Abbreviations}

GHR, growth hormone receptor; SLD, sex-linked dwarf; BMSCs, bone mesenchymal stem cells; ROS, reactive oxygen species; ATP, adenosine triphosphate; OXPHOS, oxidative phosphorylation; $\triangle \Psi \mathrm{m}$, mitochondrial membrane potential; GH, growth hormone; GHRKO, growth hormone receptor knockout; RTqPCR, reverse-transcription quantitative polymerase chain reaction 


\section{Declarations}

\section{Ethics approval and consent to participate}

All animal experiments were performed according to the protocols approved by the South China Agriculture University Institutional Animal Care and Use Committee (approval number SCAU\#0017). All animal procedures followed the regulations and guidelines established by this committee and minimized the suffering of animals.

\section{Consent for publication}

Not applicable.

\section{Availability of data and material}

All data generated or analyzed during this study are available from the corresponding author on reasonable request.

\section{Competing interests}

The authors declare that they have no competing interests.

\section{Funding}

This work was supported by grants from the Key-Area Research and Development Program of Guangdong Province (grant no. 2020B020222002), the Project of Wenshi Science Innovation Center, the Guangdong Provincial Promotion Project on Preservation and Utilization of Local Breed of Livestock and Poultry, and the China Agriculture Research System (CARS-41-G03).

\section{Author contributions}

$\mathrm{CZ}$ designed the study, wrote the manuscript, conducted the experiments, and analyzed the data. $\mathrm{BH}$ participated in the design of the study and wrote the manuscript. HW, YZ, and JL participated in data collection and interpretation and helped perform some of the experiments. WL and QN engaged in useful discussion and revised the manuscript. DZ and QL provided experimental animals. HL and XZ developed concepts, designed and supervised the study, and wrote the manuscript.

\section{Acknowledgements}

Not applicable.

\section{References}

1. Hu B, Li H, Zhang XA, Balanced Act. The effects of GH-GHR-IGF1 axis on mitochondrial function. Front Cell Dev Biol. 2021;9(Mar):1-14. 
2. Dehkhoda F, Lee CMM, Medina J, Brooks AJ. The growth hormone receptor: mechanism of receptor activation, cell signaling, and physiological aspects. Front Endocrinol (Lausanne). 2018;9(Feb):1-23.

3. Burnside J, Liou SS, Cogburn LA, Burnside J. Molecular cloning of the chicken growth hormone receptor complementary deoxyribonucleic acid: mutation of the gene in sex-linked dwarf chickens. Endocrinology. 1991;128:3183-92.

4. Guillaume J. The dwarfing gene dw: its effects on anatomy, physiology, nutrition, management. Its application in poultry industry. Worlds Poult Sci J. 1976;32:285-305.

5. Perret-Vivancos C, Abbate A, Ardail D, Raccurt M, Usson Y, Lobie PE, et al. Growth hormone activity in mitochondria depends on $\mathrm{GH}$ receptor Box 1 and involves caveolar pathway targeting. Exp Cell Res. 2006;312:215-32.

6. Brown-Borg HM, Rakoczy SG, Sharma S, Bartke A. Long-living growth hormone receptor knockout mice: potential mechanisms of altered stress resistance. Exp Gerontol. 2009;44:10-9.

7. Ploumi C, Daskalaki I, Tavernarakis N. Mitochondrial biogenesis and clearance: a balancing act. FEBS J. 2017;284:183-95.

8. Gesing A, Bartke A, Wang F, Karbownik-Lewinska M, Masternak MM. Key regulators of mitochondrial biogenesis are increased in kidneys of growth hormone receptor knockout (GHRKO) mice. Cell Biochem Funct. 2011;29:459-67.

9. Gesing A, Masternak MM, Wang F, Joseph AM, Leeuwenburgh C, Westbrook R, et al. Expression of key regulators of mitochondrial biogenesis in Growth Hormone Receptor Knockout (GHRKO) mice is enhanced but is not further improved by other potential life-extending interventions. Journals Gerontol - Ser A Biol Sci Med Sci. 2011;66(A):1062-76.

10. Liu Z, Solesio ME, Schaffler MB, Frikha-Benayed D, Rosen CJ, Werner H, et al. Mitochondrial function is compromised in cortical bone osteocytes of long-lived growth hormone receptor null mice. J Bone Miner Res. 2019;34:106-22.

11. Hu B, Hu S, Yang M, Liao Z, Zhang D, Luo Q, et al. Growth hormone receptor gene is essential for chicken mitochondrial function in vivo and in vitro. Int J Mol Sci. 2019;20:1-13.

12. Muruganandan S, Roman AA, Sinal CJ. Adipocyte differentiation of bone marrow-derived mesenchymal stem cells: cross talk with the osteoblastogenic program. Cell Mol Life Sci. 2009;66:236-53.

13. Attia N, Mashal M. Mesenchymal stem cells: the past present and future. Advances in Experimental Medicine and Biology. 2020;1312.

14. James AW. Review of signaling pathways governing MSC osteogenic and adipogenic differentiation. Scientifica (Cairo). 2013;2013:1-17.

15. Yang F, Yuan P, Hao Y, Lu Z. Emodin enhances osteogenesis and inhibits adipogenesis. BMC Complement Altern Med. 2014;14.

16. Li Y, Jin D, Xie W, Wen L, Chen W, Xu J, et al. PPAR-y and Wnt regulate the differentiation of MSCs into adipocytes and osteoblasts respectively. Curr Stem Cell Res Ther. 2017;13:185-92. 
17. Olarescu NC, Berryman DE, Householder LA, Lubbers ER, List EO, Benencia F, et al. GH action influences adipogenesis of mouse adipose tissue-derived mesenchymal stem cells. J Endocrinol. 2015;226:13-23.

18. Hsu YC, Wu YT, Yu TH, Wei YH. Mitochondria in mesenchymal stem cell biology and cell therapy: from cellular differentiation to mitochondrial transfer. Semin Cell Dev Biol. 2016;52:119-31.

19. Tormos KV, Anso E, Hamanaka RB, Eisenbart J, Joseph J, Kalyanaraman B, et al. Mitochondrial complex III ROS regulate adipocyte differentiation. Cell Metab. 2011;14:537-44.

20. Huang PL, Chen YC, Chen LH, Juan CC, Ku HH, Wang ST, et al. PGC-1 a mediates differentiation of mesenchymal stem cells to brown adipose cells. J Atheroscler Thromb. 2011;18:966-80.

21. Wang X, Hai C. Redox modulation of adipocyte differentiation: hypothesis of "redox chain" and novel insights into intervention of adipogenesis and obesity. Free Radic Biol Med. 2015;89:99-125.

22. Li H, Hu B, Luo Q, Hu S, Luo Y, Zhao B, et al. Runting and Stunting Syndrome Is Associated With Mitochondrial Dysfunction in Sex-Linked Dwarf Chicken. Front Genet. 2019;10(Jan):1-11.

23. Zhang Y, Marsboom G, Toth PT, Rehman J. Mitochondrial respiration regulates adipogenic differentiation of human mesenchymal stem cells. PLoS One. 2013;8.

24. Lin S, Li H, Mu H, Luo W, Li Y, Jia X, et al. Let-7b regulates the expression of the growth hormone receptor gene in deletion-type dwarf chickens. BMC Genom. 2012;13:306.

25. Agarwal SK, Cogburn LA, Burnside J. Comparison of gene expression in normal and growth hormone receptor-deficient dwarf chickens reveals a novel growth hormone regulated gene. Biochem Biophys Res Commun. 1995;206:153-60.

26. Li H, Hu B, Hu S, Luo W, Sun D, Yang M, et al. High expression of BCL6 inhibits the differentiation and development of hematopoietic stem cells and affects the growth and development of chickens. $J$ Anim Sci Biotechnol. 2021;12:1-13.

27. Hardouin P, Pansini V, Cortet B. Bone marrow fat. Jt Bone Spine. 2014;81:313-19.

28. Duque G. Bone and fat connection in aging bone. Curr Opin Rheumatol. 2008;20:429-34.

29. Nuttall ME, Shah F, Singh V, Thomas-Porch C, Frazier T, Gimble JM. Adipocytes and the regulation of bone remodeling: a balancing act. Calcif Tissue Int. 2014;94:78-87.

30. Gimble JM, Robinson CE, Wu X, Kelly KA, Rodriguez BR, Kliewer SA, et al. Peroxisome proliferatoractivated aeceptor- $\gamma$ activation by thiazolidinediones induces adipogenesis in bone marrow stromal cells. Mol Pharmacol. 1996;50:1087-94.

31. Zhao XY, Chen XY, Zhang ZJ, Kang Y, Liao WM, Yu WH, et al. Expression patterns of transcription factor PPARY and C/EBP family members during in vitro adipogenesis of human bone marrow mesenchymal stem cells. Cell Biol Int. 2015;39:457-65.

32. Hofmann AD, Beyer M, Krause-Buchholz U, Wobus M, Bornhäuser M, Rödel G. Oxphos supercomplexes as a hallmark of the mitochondrial phenotype of adipogenic differentiated human MSCS. PLoS One. 2012;7. 
33. Madamanchi NR, Runge MS. Mitochondrial dysfunction in atherosclerosis. Circ Res. 2007;100:46073.

34. Guzik TJ, Harrison DG. Vascular NADPH oxidases as drug targets for novel antioxidant strategies. Drug Discov Today. 2006;11:524-33.

35. Higuchi M, Dusting GJ, Peshavariya H, Jiang F, Hsiao ST-F, Chan EC, et al. Differentiation of human adipose-derived stem cells into fat involves reactive oxygen species and forkhead Box 01 mediated upregulation of antioxidant enzymes. Stem Cells Dev. 2013;22:878-88.

36. Zorova LD, Popkov VA, Plotnikov EY, Silachev DN, Pevzner IB, Jankauskas SS, et al. Mitochondrial membrane potential. Anal Biochem. 2018;552:50-9.

37. Skulachev VP. Role of uncoupled and non-coupled oxidations in maintenance of safely low levels of oxygen and its one-electron reductants. Q Rev Biophys. 1996;29:169-202.

38. Rajasekaran NS, Connell P, Christians ES, Yan LJ, Taylor RP, Orosz A, et al. Human alpha B-crystallin mutation causes oxido-reductive stress and protein aggregation cardiomyopathy in mice. Cell. 2007;130:427-39.

39. Cunha M, Caldeira da Silva FC, Cerqueira CM, Kowaltowski FJ. A. Mild mitochondrial uncoupling as a therapeutic strategy. Curr Drug Targets. 2011;12:783-89.

40. Popov LD. Mitochondrial biogenesis: an update. J Cell Mol Med. 2020;24:4892-99.

41. Hao Z, Wu T, Cui X, Zhu P, Tan C, Dou X, et al. N6-Deoxyadenosine methylation in mammalian mitochondrial DNA. Mol Cell. 2020;78:382-95.e8.

42. Zhuang $H$, Zhang $X$, Zhu C, Tang X, Yu F, Shang G, et al. Molecular mechanisms of PPAR- $Y$ : governing MSC osteogenic and adipogenic differentiation. Curr Stem Cell Res Ther. 2016;11:255-64.

\section{Tables}

Table 1 Primer sequences in reverse-transcription quantitative PCR. 


\begin{tabular}{|c|c|c|c|}
\hline Genes & Primer sequences, $5^{\prime}-3^{\prime}$ & Temperature, ${ }^{\circ} \mathrm{C}$ & Size, bp \\
\hline \multirow[t]{2}{*}{ GHR } & GCAAGTGCAGGTCACCTGAG & 56 & 153 \\
\hline & CCGGACATTCTTTCCAGTCT & & \\
\hline \multirow[t]{2}{*}{ ND1 } & ACCCAAGAGCCCATCTACCT & 56 & 154 \\
\hline & GTCCGGCGGCATATTCTACA & & \\
\hline \multirow[t]{2}{*}{ ND2 } & CCGAGCGATTGAAGCCACTA & 56 & 103 \\
\hline & TCATTGTCCGGTGGATCAGG & & \\
\hline \multirow[t]{2}{*}{ CYTB } & CAGCAGACACATCCCTAGCC & 56 & 104 \\
\hline & GAAGAATGAGGCGCCGTTTG & & \\
\hline \multirow[t]{2}{*}{$\operatorname{cox} 1$} & ACTACTTACCGACCGCAACC & 56 & 132 \\
\hline & CCGAAACCTGGGAGGATGAG & & \\
\hline \multirow[t]{2}{*}{$\operatorname{cox} 2$} & TCGGGGTAAAAACAGACGCA & 56 & 70 \\
\hline & АCTCCTGGTCGAGTGGTGAT & & \\
\hline \multirow[t]{2}{*}{ ATP6 } & TACAGCCACAATCGCCCTAC & 56 & 123 \\
\hline & AGGACGAAGACGTAGGCTTG & & \\
\hline \multirow[t]{2}{*}{ ATP8 } & AACCCAAACCCATGATTCTCCA & 56 & 139 \\
\hline & AGGTTCAGGGGGTGGGTTTA & & \\
\hline \multirow[t]{2}{*}{ PGC1a } & TCCTTTCCTCAACGCAGGTC & 56 & 153 \\
\hline & TCTTGCACGTGAGGGAGAAC & & \\
\hline \multirow[t]{2}{*}{ NRF1 } & ACGAGGACTCACСTTCCTCA & 56 & 163 \\
\hline & TGTGGTCGCTTCCGTTTCTT & & \\
\hline \multirow[t]{2}{*}{ TFAM } & GACCTCGAAGTGGCTTCAAC & 56 & 144 \\
\hline & GAGCAAGCTGAAGGTATGGCT & & \\
\hline \multirow[t]{2}{*}{ PPARY } & CCAGCGACATCGACCAGTTA & 60 & 275 \\
\hline & AGAGCGAAACTGACATCGCT & & \\
\hline \multirow[t]{2}{*}{$C E B P / a$} & GACAAGAACAGCAACGAGTACC & 60 & 195 \\
\hline & CCTGAAGATGCCCCGCAGAGT & & \\
\hline$C E B P / \beta$ & $\begin{array}{l}\text { AACCTGTCCACCTCGTCCT } \\
\text { CCAAGACTTTGTGCTGCGTC }\end{array}$ & 60 & 241 \\
\hline$\beta$-actin & GATATTGCTGCGCTCGTTG & 56 & 178 \\
\hline
\end{tabular}


Table 2 Oligonucleotide sequence in this study.

\begin{tabular}{|ll|}
\hline Fragment name & Sequence, $5^{\prime}-3^{\prime}$ \\
\hline si-GHR & CCUCGAUUUGGAUACCAUA \\
\hline
\end{tabular}

\section{Figures}
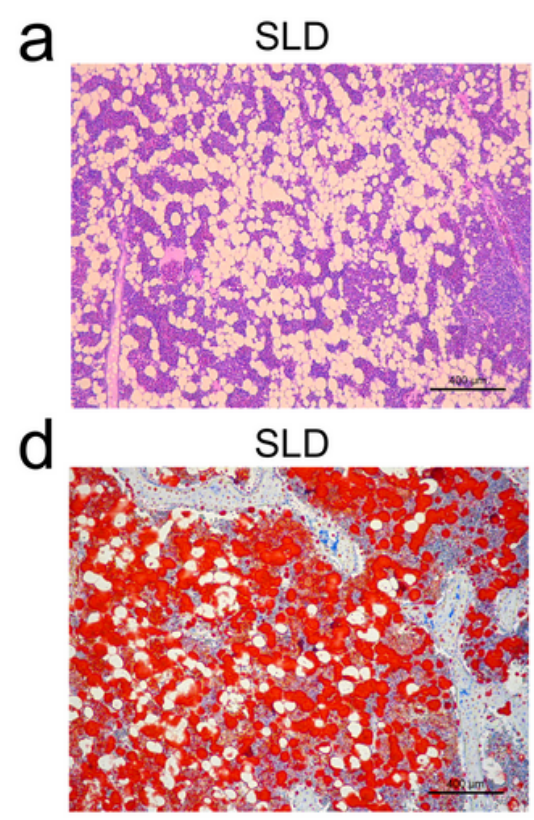

9

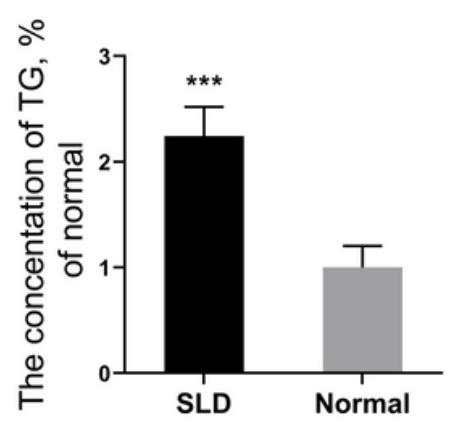

b

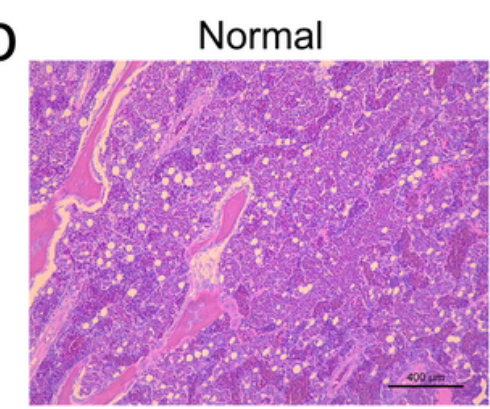

e

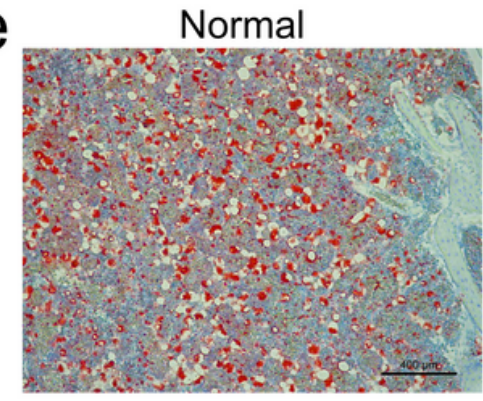

$\mathrm{h}$

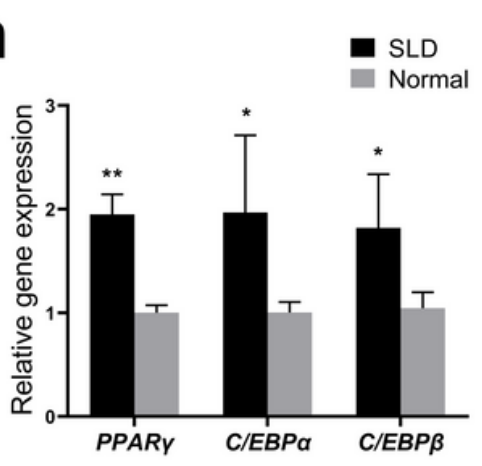

C

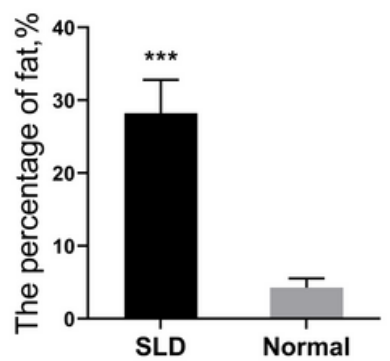

f

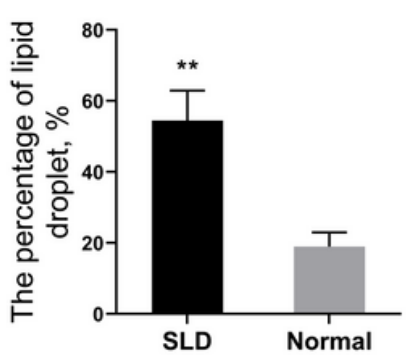

i

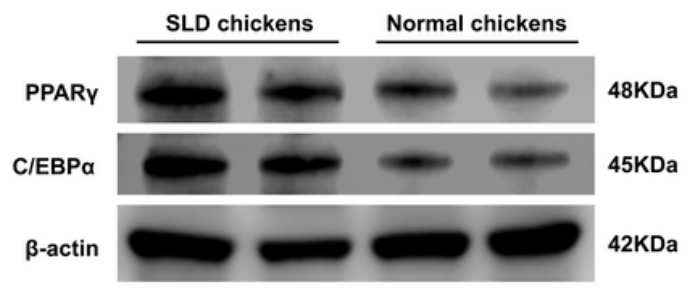

Figure 1

Fat deposition and differentiation of bone mesenchymal stem cells in 21-d-old sex-linked dwarf (SLD) and normal chickens. $a$ and $b$ Hematoxylin and eosin-stained paraffin sections of bone marrow tissue from SLD and normal chickens. Scale bar $=400 \mu \mathrm{m}$. c The percentage of fat in volume (\%) in bone marrow tissue from SLD and normal chickens. $d$ and e Oil red O-stained frozen sections of bone marrow 
tissue from SLD and normal chickens. Scale bar $=400 \mu \mathrm{m}$. $\mathrm{f}$ The percentage of lipid droplets in volume (\%) in bone marrow tissue from SLD and normal chickens. g The triglyceride (TG) levels (\% of normal) in bone marrow tissues of SLD and normal chickens. $h$ Expression of genes related to adipogenic differentiation in bone mesenchymal stem cells, as determined by reverse-transcription Qpcr. i Protein levels related to adipogenic differentiation in bone mesenchymal stem cells, as determined by western blot. $\beta$-actin was used as the control. Data are expressed as the mean $\pm S E M$. ${ }^{\star} P<0.05$, ${ }^{\star \star P} P<0.01, \star \star \star P<$ 0.001

\section{a}

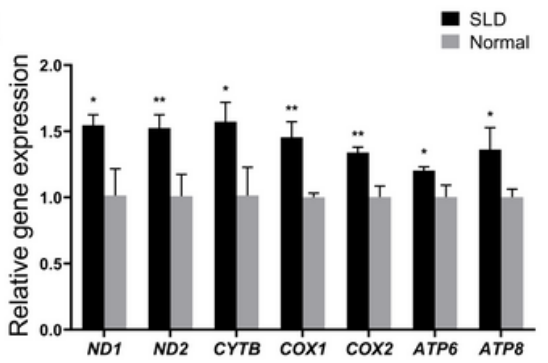

d

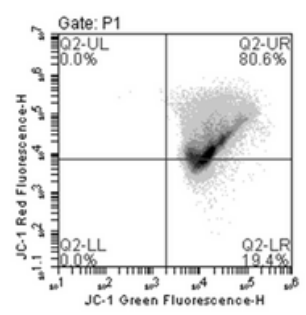

b

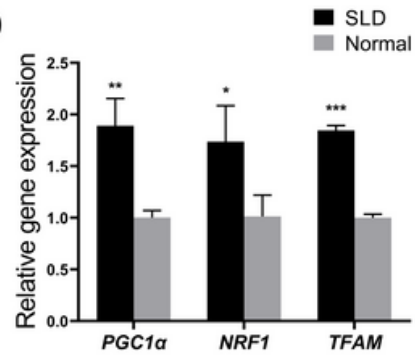

e
C

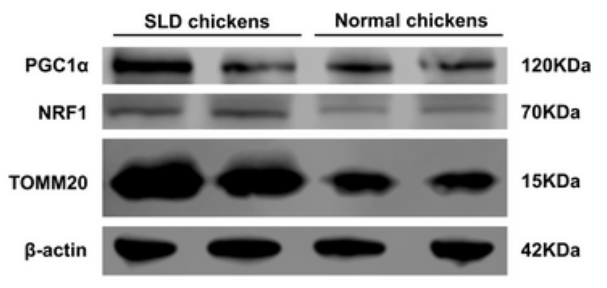

$\mathrm{f}$
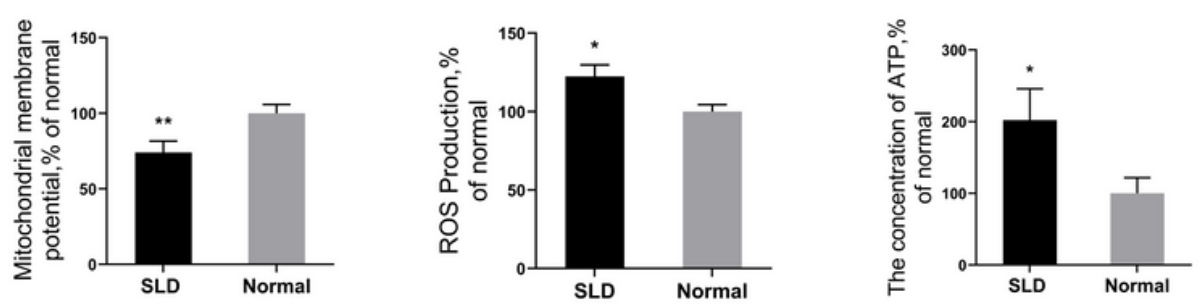

Figure 2

Mitochondrial function in bone mesenchymal stem cells of 21-d-old sex-linked dwarf (SLD) and normal chickens. a Expression of genes related to mtDNA-encoded oxidative phosphorylation in bone mesenchymal stem cells (BMSCs), as determined by reverse-transcription (RT) qPCR. b Expression of genes related to mitochondrial biogenesis in BMSCs, as determined by RT-qPCR. c Protein levels of mitochondrial biogenesis and mitochondrial marker genes in BMSCs by western blot. $\beta$-actin was used as the control. $d$ Mitochondrial membrane potential in BMSCs. Red fluorescence represents aggregation of $\mathrm{JC}-1$, green fluorescence represents monomeric $\mathrm{JC}-1, \Delta \Psi \mathrm{m}$ was represented as the ratio of aggregated and monomeric JC-1. e Reactive oxygen species (ROS) levels in BMSCs. $f$ The ATP levels in BMSCs. Data are expressed as the mean \pm SEM. ${ }^{*} P<0.05$, ${ }^{\star *} P<0.01$, $* \star * P<0.001$ 
a

b

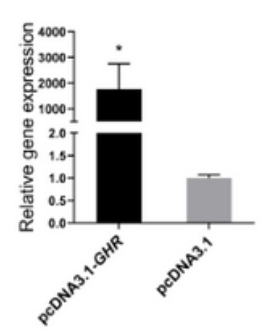

e
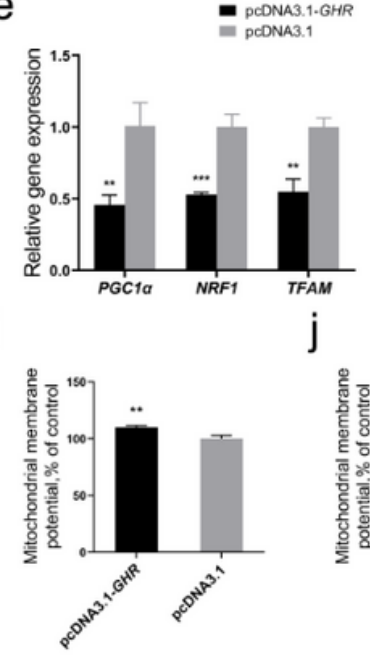

C

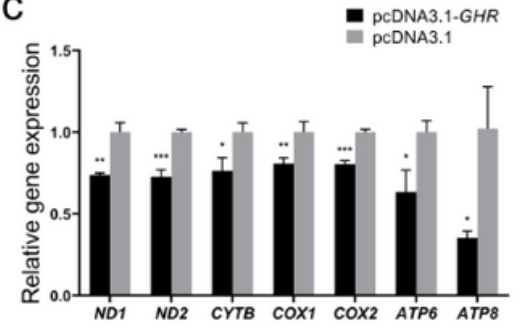

d

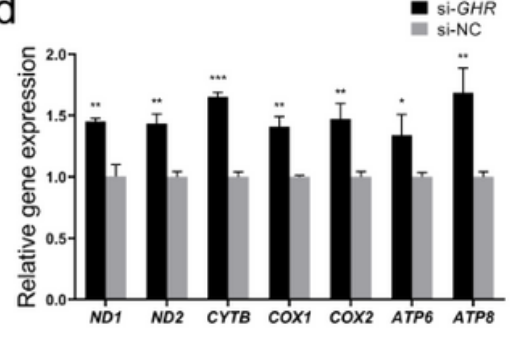

f

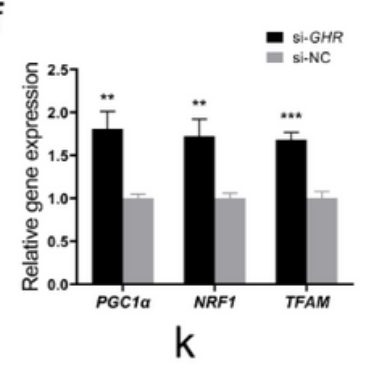

g

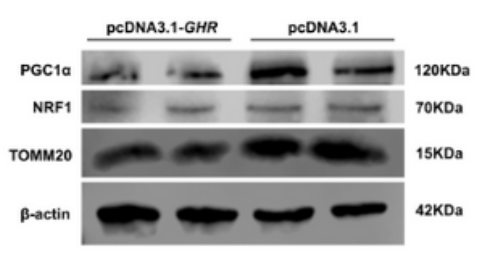

I

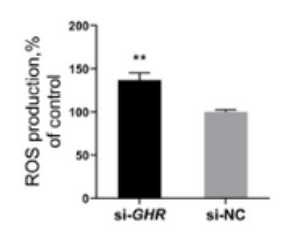

m h
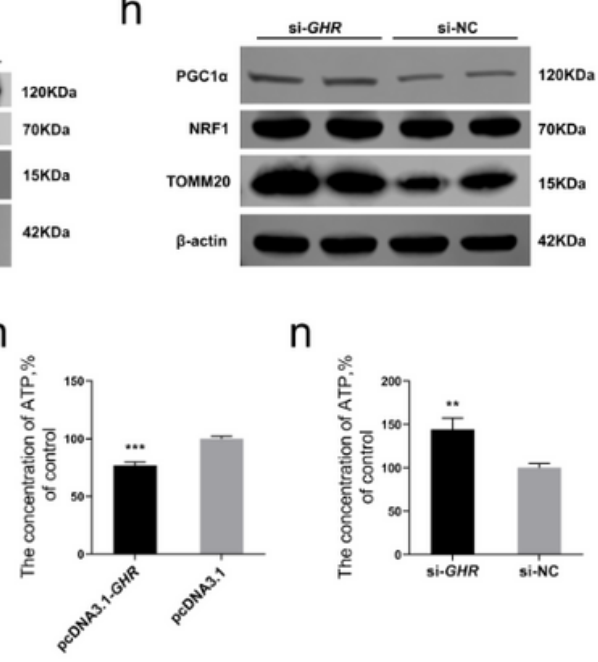

$\mathrm{n}$

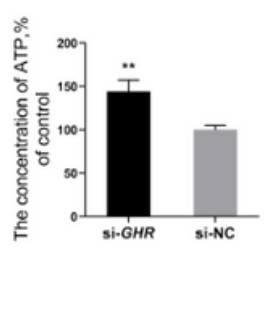

Figure 3

Overexpression and knockdown of growth hormone receptor (GHR) and effects on mitochondria in differentiated bone mesenchymal stem cells. a and b Expression of GHR in bone mesenchymal stem cells (BMSCs) after being transfected with an overexpression vector or siRNA. After overexpression and knockdown of GHR: $c$ and d expression of genes related to mtDNA-encoded ox idative phosphorylation in BMSCs; e and f expression of genes related to mitochondrial biogenesis in BMSCs; $g$ and $h$ protein levels of PGC1a, NRF1, and TOMM20; i and j mitochondrial membrane potential in BMSCs; $k$ and I reactive oxygen species (ROS) production in BMSCs; $m$ and $n$ the concentration of ATP in BMSCs. Data are expressed as the mean \pm SEM. ${ }^{*} P<0.05,{ }^{*} \mathrm{P}<0.01,{ }^{*} * \mathrm{P}<0.001$ 
a

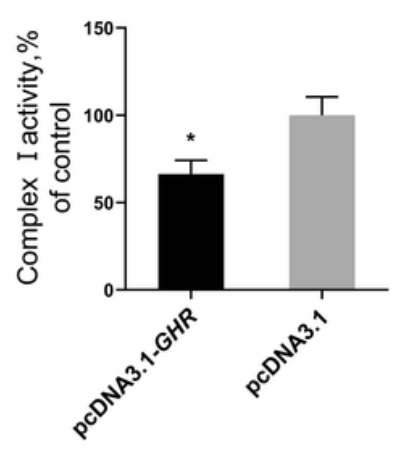

e

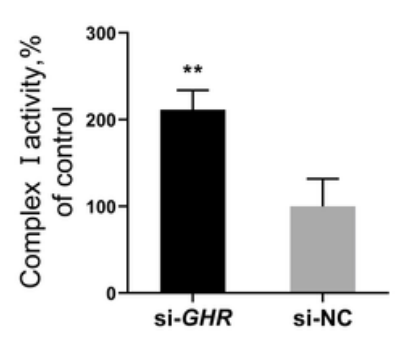

b

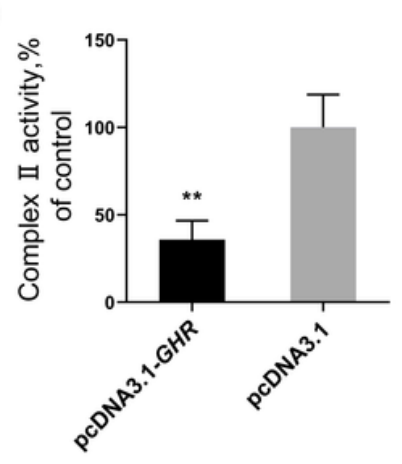

f

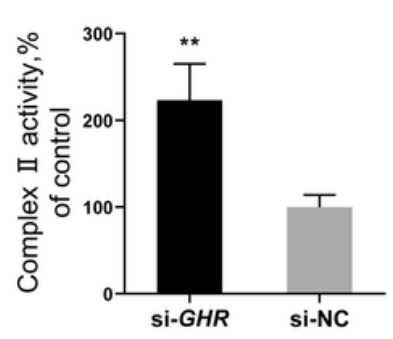

C

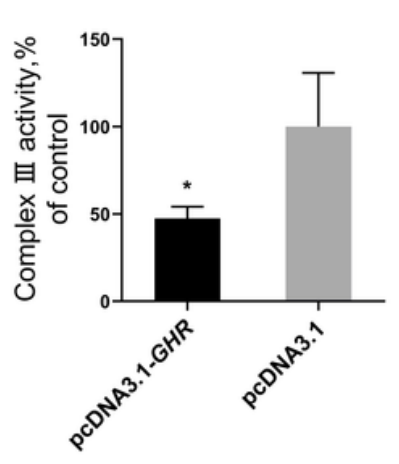

g

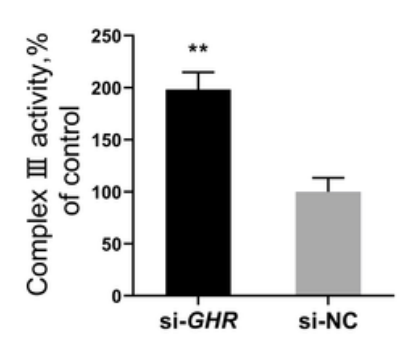

d

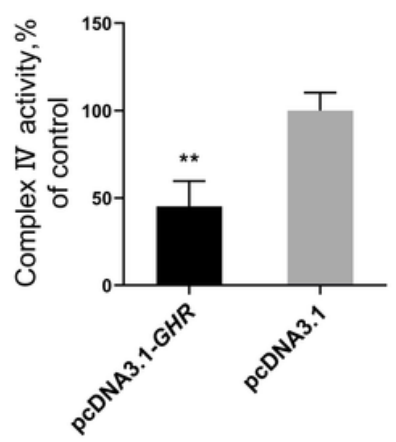

h

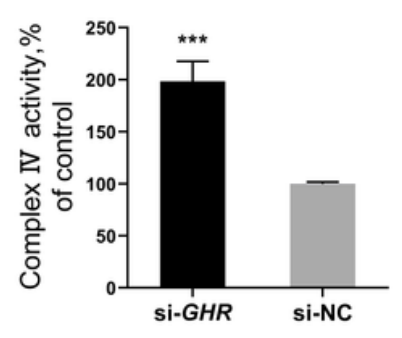

\section{Figure 4}

Overexpression and knockdown of GHR and effects on enzymatic activity of oxidative phosphorylation complexes in bone mesenchymal stem cells. After overexpression of GHR, enzymatic activity of a complex I, b complex II, c complex III, and d complex IV in bone mesenchymal stem cells. After knockdown of GHR, enzymatic activity of e complex I, f complex II, $\mathrm{g}$ complex III, and h complex IV in bone mesenchymal stem cells. Data are expressed as the mean \pm SEM. ${ }^{*} P<0.05,{ }^{\star} * P<0.01,{ }^{\star} * \star P<0.001$ 
a
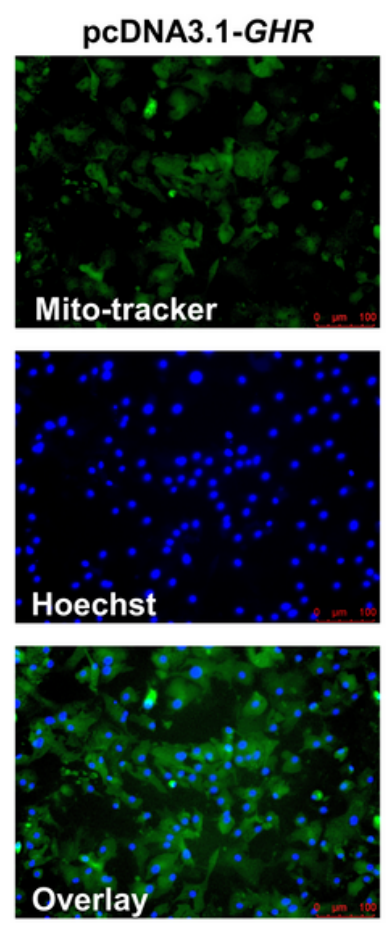

C

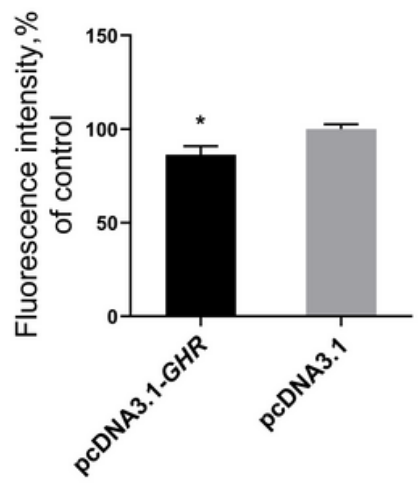

b

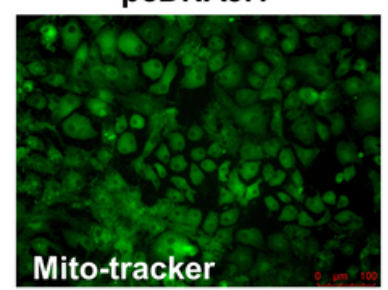

Hoechst

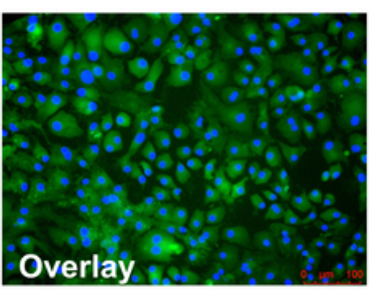

si-GHR
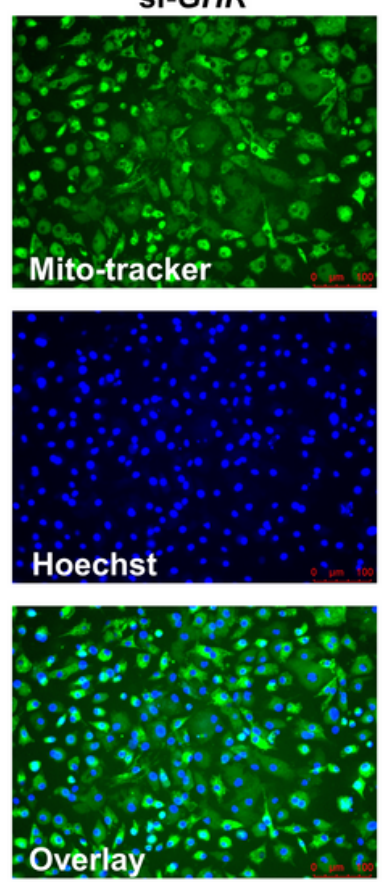

si-NC

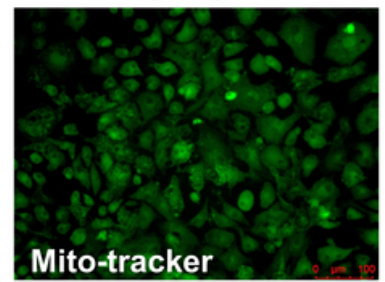

Hoechst

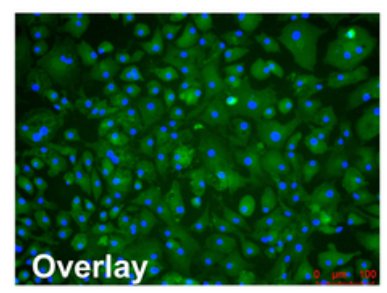

d

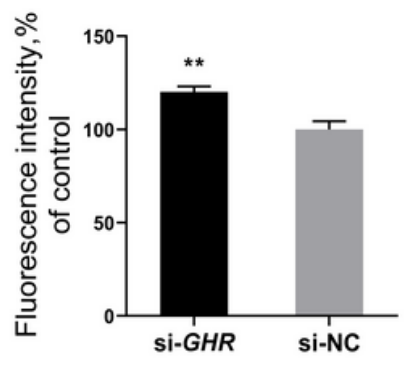

\section{Figure 5}

Overexpression and knockdown of growth hormone receptor (GHR) and effects on mitochondrial number and quality in bone mesenchymal stem cells. After overexpression and knockdown of GHR: a and b Mitotracker staining of differentiated BMSCs and $\mathrm{c}$ and d fluorescence intensity of Mito-tracker staining. Data are expressed as the mean \pm SEM. ${ }^{\star} \mathrm{P}<0.05,{ }^{\star *} \mathrm{P}<0.01$ 
a

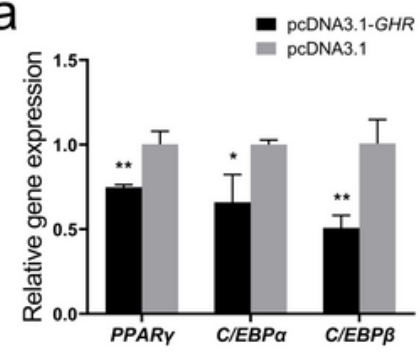

C

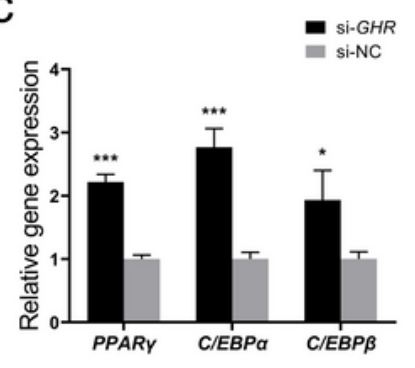

e

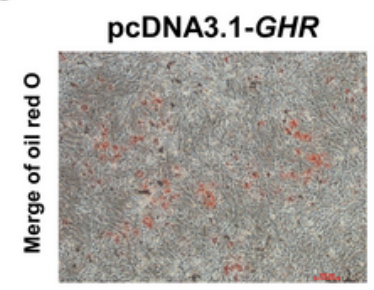

g

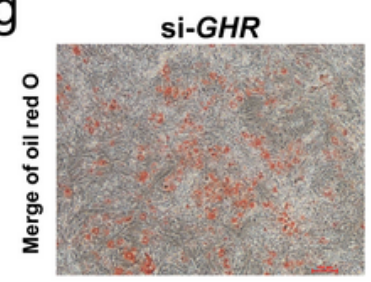

i

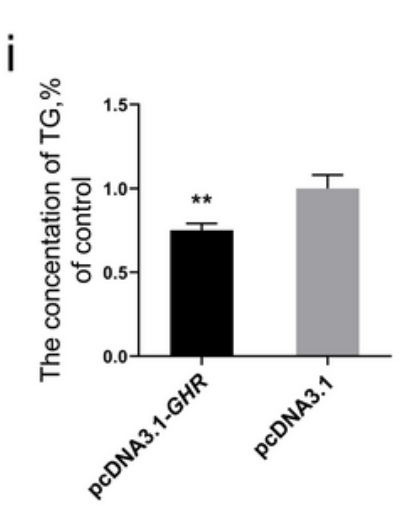

b

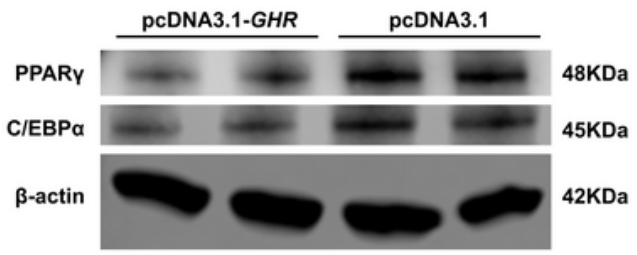

d

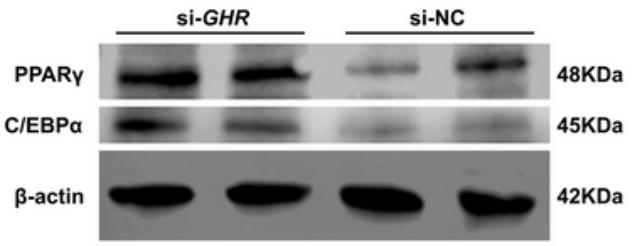

si-NC
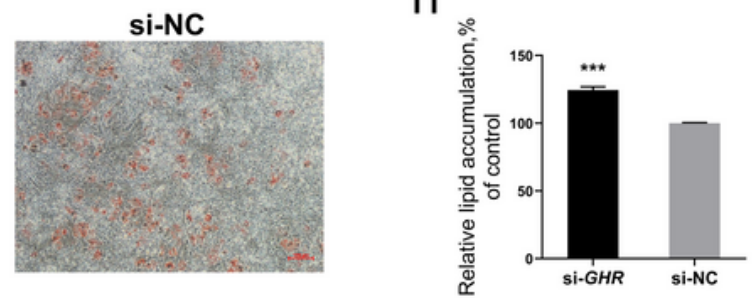

f

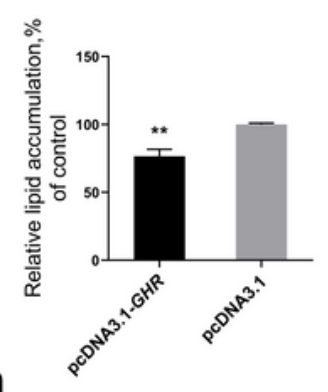

pcDNA3.1

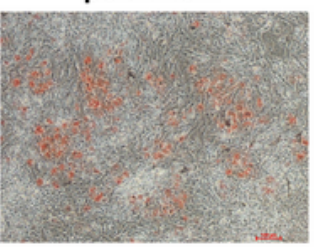

h

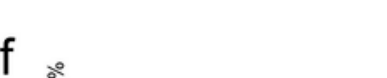

j

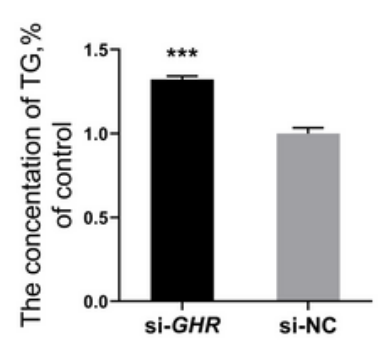

\section{Figure 6}

Overexpression and knockdown of growth hormone receptor (GHR) and effects on adipogenic differentiation of bone mesenchymal stem cells. After overexpression of GHR: a expression of genes associated with adipogenic differentiation in BMSCs and b protein levels of adipogenic differentiation genes. After knockdown of GHR: c expression of genes associated with adipogenic differentiation in BMSCs and d protein levels of adipogenic differentiation genes. $\mathrm{e}-\mathrm{h}$ Oil red $\mathrm{O}$ test and lipid droplet 
quantification in BMSCs differentiated for $5 \mathrm{~d}$ after overexpression and knockdown of GHR. Scale bars $=100 \mu \mathrm{m} . \mathrm{i}$ and $\mathrm{j}$ The triglyceride (TG) contents in BMSCs after overexpression and knockdown of GHR. Data are expressed as the mean \pm SEM. ${ }^{*} \mathrm{P}<0.05,{ }^{*} \mathrm{P}<0.01,{ }^{\star \star *} \mathrm{P}<0.001$ 